\title{
Discovery of cancer driver genes based on nucleotide context
}

Felix Dietlein 1,2,6*, Donate Weghorn ${ }^{3,4,6}$, Amaro Taylor-Weiner ${ }^{1,2}$, André Richters ${ }^{2,5}$, Brendan Reardon 1,2 , David Liu1,2, Eric S. Lander², Eliezer M. Van Allen 1,2,7* \& Shamil R. Sunyaev3,4,7*

${ }^{1}$ Department of Medical Oncology, Dana-Farber Cancer Institute, Harvard Medical School, Boston, MA 02215, USA.

${ }^{2}$ Broad Institute of Massachusetts Institute of Technology and Harvard, Cambridge, MA, 02142, USA. 3Division of Genetics, Brigham and Women's Hospital, Harvard Medical School, Boston, MA 02115, USA. ${ }^{4}$ Department of Biomedical Informatics, Harvard Medical School, Boston, MA 02115, USA.

${ }^{5}$ Koch Institute for Integrative Cancer Research, Massachusetts Institute of Technology, Cambridge, MA 02139, USA.

${ }^{6}$ These authors contributed equally: Felix Dietlein, Donate Weghorn.

7These authors jointly supervised this work: Eliezer M. Van Allen, Shamil R. Sunyaev.

*email: ssunyaev@rics.bwh.harvard.edu; EliezerM_VanAllen@dfci.harvard.edu;

Felix_Dietlein@dfci.harvard.edu

Many cancer genomes contain large numbers of somatic mutations, but few of these mutations drive tumor development. Current approaches to identify cancer driver genes are largely based on mutational recurrence, i.e. they search for genes with an increased number of nonsynonymous mutations relative to the local background mutation rate. Multiple studies have noted that the sensitivity of recurrence-based methods is limited in tumors with high background mutation rates, because passenger mutations dilute their statistical power. Here, we observe that passenger mutations tend to occur in characteristic nucleotide sequence contexts, while driver mutations follow a different distribution pattern determined by the location of functionally relevant genomic positions along the protein-coding sequence. To discover new cancer genes, we searched for genes with an excess of mutations in unusual nucleotide contexts that deviate from the characteristic context around passenger mutations. By applying this statistical framework to wholeexome sequencing data from 12,004 tumors, we discovered a long tail of novel candidate cancer genes with mutation frequencies as low as $1 \%$ and functional supporting evidence. Our results show that considering both the number and the nucleotide context around mutations helps identify novel cancer driver genes, particularly in tumors with high background mutation rates. 
24 Multiple algorithms have been developed to systematically identify genes that drive tumor

25 formation ${ }^{1-5}$. Most search for genes harboring more nonsynonymous mutations than expected

26 based on the local background mutation rate ${ }^{1-5}$. These recurrence-based methods have

27 successfully identified many novel cancer genes $4,6,7$. However, several studies have noted that the

28 sensitivity of recurrence-based approaches is limited $6,8,9$, because functionally neutral passenger

29 mutations dilute their statistical power to detect recurrent driver mutations ${ }^{10,11}$. Hence, due to the

30 high prevalence of passenger mutations in tumors with high background mutation rates, recent

31 studies have concluded that orders of magnitude more sequencing data would be needed to

32 establish a comprehensive catalog of all cancer driver genes using recurrence-based methods ${ }^{6,8,9}$

33 (Fig. S1).

34 Passenger mutations are not uniformly distributed along the cancer genome. Rather, they are 35 enriched within characteristic nucleotide sequence contexts, whose specificity depends on the 36 specific mutational processes active in a given tumor ${ }^{12-15}$. For instance, APOBEC enzymes scan 37 single-stranded DNA for specific nucleotide sequence motifs and deaminate cytidine to uracile 38 within these motifs ${ }^{16-18}$. Similarly, mutant polymerase $\varepsilon$ randomly introduces passenger mutations 39 in a non-uniform manner, since its fidelity depends strongly on the local nucleotide context ${ }^{19-22}$. 40 For driver mutations, the distribution depends not only on the local nucleotide context, but also on 41 the location of functionally relevant positions along the protein sequence ${ }^{23-26}$. Thus, we 42 hypothesized that the nucleotide context would differ substantially around driver and passenger 43 mutations.

44 Based on this hypothesis, we here developed a biologically informed statistical approach for 45 discovering new cancer genes. Briefly, our approach searches for genes harboring an excess of 46 mutations in unusual nucleotide contexts that deviate from the characteristic nucleotide context 47 around passenger mutations (Fig. 1a, Methods).

48 Our new method requires modeling the nucleotide context around passenger mutations. The 5' 49 and 3' nucleotides immediately adjacent to a passenger mutation have the strongest effect on the

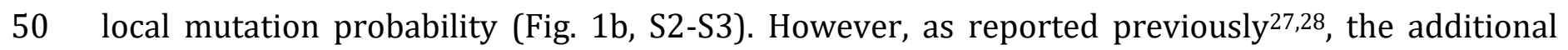
51 upstream and downstream nucleotides flanking a passenger mutation also influence its mutation 52 probability substantially (Fig. 1b, S2-S3). Traditionally, the effect of the flanking 5' and 3' 53 nucleotides on the local mutation probability has been modeled by determining the mutation 54 probabilities of all possible trinucleotide contexts independently ${ }^{12-15}$. As the number of flanking 
55 nucleotides increases, the number of possible sequence contexts grows exponentially - soon 56 exceeding the number of mutations per tumor (Fig. S4). Hence, it is no longer feasible to analyze 57 all possible sequence contexts independently.

58 Instead, we approximated the context-specific mutation probability by assuming that each 59 flanking nucleotide contributed independently and multiplicatively to the local mutation 60 probability (Fig. 1c-f, S5-S8, Methods). For instance, we approximated the mutation probabilities 61 of trinucleotide contexts as products of the effects of their flanking 5' and 3' nucleotides, as well as 62 their base substitution type (Fig. 1c-d, S7a). We developed a composite likelihood model ${ }^{29}$ to 63 extend this approach to larger nucleotide contexts (Fig. 1e). This model closely matched the 64 observed mutation probabilities for the 29 cancer types examined in this study (Fig. 1e-f, S7b-c). 65 Although the immediately adjacent 5' and 3' nucleotides had the strongest impact on the local 66 mutation probability, also flanking nucleotides outside of the trinucleotide context had a 67 substantial effect in this composite likelihood model, thus refining our approximation of the local 68 mutation probabilities (Fig. S7d, S8).

69 We then examined whether the composite likelihood model could distinguish driver from 70 passenger mutations using 10 established melanoma genes and 5 non-cancer-related genes that 71 had been reported as false-positive findings in previous cancer gene discovery studies ${ }^{3}$ (Fig. 2). 72 While mutations in non-cancer-related genes closely followed the expected context-dependent 73 distribution pattern derived from the composite likelihood model, most mutations in cancer genes 74 fell in nucleotide contexts that deviated from the expectation of the model. This suggested that 75 considering the broad nucleotide context around mutations could indeed provide new biological 76 information to help distinguish between driver and passenger mutations.

77 Encouraged by these observations, we developed a statistical framework to detect cancer driver 78 genes that considers both mutation counts and nucleotide contexts. In our model, the probability 79 of observing the number $n_{g}$ and the context-dependent distribution $v_{g}$ of nonsynonymous 80 mutations in a gene $g\left(P\left(n_{g}, v_{g} \mid s_{g} ; \lambda_{g}\right)\right)$ depends on the number of synonymous mutations $s_{g}$ and 81 the context-specific mutation rates $\lambda_{g}$. We decomposed this probability into the probability of 82 observing $n_{g}$ nonsynonymous mutations, given the number of synonymous mutations

$83 s_{g}$ ("mutation count"; $P\left(n_{g} \mid s_{g}\right)$ ), and the probability of these $n_{g}$ nonsynonymous mutations falling 
84 in nucleotide contexts $v_{g}$, given their context-specific mutation rates $\lambda_{g}$ ("nucleotide context"; $\left.85 P\left(v_{g} \mid n_{g} ; \lambda_{g}\right)\right)$ :

$$
P\left(n_{g}, v_{g} \mid s_{g} ; \lambda_{g}\right):=\underbrace{P\left(n_{g} \mid s_{g}\right)}_{\text {mutation count }} \cdot \underbrace{P\left(v_{g} \mid n_{g} ; \lambda_{g}\right)}_{\text {nucleotide context }}
$$

87 Here, $P\left(n_{g} \mid s_{g}\right)$ reflects the established statistics used by existing recurrence-based methods for 88 cancer gene discovery ${ }^{1-5}$. The $p$-value of $P\left(v_{g} \mid n_{g} ; \lambda_{g}\right)$ was derived by comparing the observed nucleotide contexts $v_{g}$ against a large number of random scenarios generated by a Monte Carlo simulation approach based on the same the context-specific mutation rates $\lambda_{g}{ }^{30,31}$. As shown by

91 Q-Q-plots 32 , the $p$-values derived from $P\left(n_{g} \mid s_{g}\right), P\left(v_{g} \mid n_{g} ; \lambda_{g}\right)$, and $P\left(v_{g}, n_{g} \mid s_{g} ; \lambda_{g}\right)$ closely 92 approximated a uniform distribution, which indicated that our models were reasonably well 93 calibrated to the observed data (Fig. 3a, Methods).

94 Notably, mutational count and nucleotide context provided complementary criteria for detecting 95 cancer genes (Fig. 3a). In cancer types with low background mutation rates, such as thyroid 96 cancer, mutational counts were highly informative. In cancer types with high background mutation rates, such as melanoma, the nucleotide context was the dominant criterion. Combining both criteria identified several candidate cancer genes that could not be identified based on mutational count or nucleotide context alone (Fig. 3a). We applied our statistical framework to whole-exome sequencing data from 12,004 individual tumors spanning 29 different tumor types (Fig. S9, Table S1). The results of these analyses are summarized here (Fig. 3-4, S9-45) and at www.cancer-genes.org, for various false-discovery rate (FDR) thresholds. For FDR<0.25, we identified 697 gene-tumor pairs, i.e. pairs of significantly mutated genes and their associated tumor type. These gene-tumor pairs involved 379 distinct genes, with 423 gene-tumor pairs being novel. The corresponding numbers were 484, 252 and 231 for FDR<0.05, as well as 395, 201, and 168 for FDR<0.01 (Tables 1, S2-S3). Gene-tumors pairs were considered novel if they were not reported as significantly mutated in at least two computational studies, among all TCGA marker papers, a meta-analysis of 876 publications, and two large-scale pan-cancer gene discovery studies ${ }^{4,6,7,33}$.

111 the novel gene-tumor pairs (49\%) involved canonical cancer genes in the Cancer Gene Census ${ }^{34,35}$, 112 compared with a rate of $3.8 \%$ for random gene-tumor pairs. We systematically reviewed the 
113 literature to further investigate the experimental or clinical support for of the novel gene-tumor

114 pairs. We only considered publications with experimental data supporting the causal involvement

115 of these genes in carcinogenesis and excluded functionally unsupported reports of mutations

116 (Methods). A majority of the novel gene-tumor pairs (82\%) had experimental support, with 61\%

117 having support in the same tumor type, in which we detected them as significantly mutated (Fig.

$1183 \mathrm{~b}$, Tables 1, S2-S3). In contrast, the rate for random gene-tumor pairs was 17\%. Overall, 11\%

119 (75/697, FDR<0.25), 6\% (30/484, FDR<0.05), and 4\% (16/395, FDR<0.01) of the significant gene-

120 tumor pairs had no literature support, which is roughly in accordance with these FDR thresholds

121 (Fig. 3b, Tables 1, S2-S3).

122 We asked whether considering the nucleotide context identified candidate cancer genes that were

123 not discovered based on recurrence alone. Among gene-tumor pairs previously reported as

124 significantly mutated, 74\% were also identified by using an established recurrence-based

125 approach $^{3}$ (FDR<0.25 for both methods, Fig. 3c-d, S10). In contrast, among novel gene-tumor

126 pairs, only 33\% were identified based on recurrence alone. In particular, our statistical framework

127 identified numerous biologically relevant candidate cancer genes that were not identified based

128 on recurrence alone. For instance, HDAC4 (histone deacetylase 4) was significantly mutated in

129 gastroesophageal cancer $\left(F D R=5.5 \times 10^{-2}\right.$ by nucleotide context and recurrence; FDR $=6.8 \times 10^{-1}$ by

130 recurrence alone; not reported as significant previously; Fig. 3e, S11). Histone deacetylases have

131 been implicated in tumor formation ${ }^{36-38}$ and HDAC4 displayed two mutational hotspots: gastric

132 cancers with disruptive frameshift mutations (P901fs), and esophageal cancers with recurrent

133 missense mutations (F746L) (Fig. 3e). Similarly, we identified POLR2A (RNA polymerase II subunit

$134 \mathrm{~A})$ as significantly mutated in lung adenocarcinoma (FDR=1.07 $\times 10^{-5}$ by nucleotide context and

135 recurrence; FDR=1.0 by recurrence; not reported as significant previously; Fig. 4, S12). Mutations

136 in POLR2A have been implicated in the development of meningioma ${ }^{39}$, and POLR2A has been

137 identified as a therapeutic target in colon cancer due to its frequent co-deletion with TP5340.

138 Further, we noticed that POLR2A contained recurrent mutations in positions that are relevant for

139 the protein-DNA interaction (Fig. S12). Additional biologically relevant candidate cancer genes

140 that were not identified based on recurrence included ANAPC1, FGFR4, IKZF3, PARG, SOX17, and

$141 Z F X(F D R<0.1$ by nucleotide context and recurrence; FDR=1.0 by recurrence; Fig. 4, S11-S12,

142 Tables S2-S3). In addition, we observed that the following cancer-related signaling complexes

143 contained several candidate cancer genes, i.e. new cancer genes or gene-tumor pairs: modulation 
144 of Ras signaling ( $R H O A, R H O B, R R A S 2)$, cell cycle regulation (CCNQ, CDK4), regulation of protein 145 levels (EEF1A1, EIF1AX, MIA2), the catenin/cadherin complex (FAT1, FAT3, FAT4), DNA 146 polymerases (POLQ, POLR2A, REV3L), regulation of transcription (MAML2, SF3B2), modulation of 147 apoptosis ( $A C V R 2 A, A C V R 1 B, C A S P 8, B I R C 3, B I R C 6)$, and epigenetic modification (Fig. S13). In these 148 signaling complexes, 64\% (118/183) of the gene-tumor pairs had not been reported as 149 significantly mutated previously, and 60\% (110/183) of the gene-tumor pairs were not identified 150 by recurrence alone (Fig. 4).

151 Taken together, our findings demonstrate that characterization of the broad nucleotide context 152 around somatic passenger mutations enhances cancer gene discovery, particularly in tumor types 153 with high background mutation rates. Consideration of the nucleotide context for cancer gene 154 discovery does not require prior knowledge of the location of functionally relevant positions or 155 the biological effect of mutations. Hence, nucleotide contexts may ultimately be amenable to 156 variant and gene discovery in non-coding regions of the cancer genome. Through our statistical 157 model we identified a long tail of reasonable candidate cancer genes that may form the foundation 158 for future experimental and clinical studies. The new statistical framework is available as a fully 159 executable software tool called MutPanning (www.cancer-genes.org) and can be executed online 160 as a module on the GenePattern platform ${ }^{41}$ (www.genepattern.org).

\section{Acknowledgments}

163 We thank Dr. Gad Getz for valuable comments and suggestions. F.D. was supported by EMBO 164 (ALTF 502-2016). E.M.V.A. and S.R.S received funding from the National Institutes of Health (K08 165 CA188615, R01 CA227388 to E.M.V.A; R01 MH101244, R35 GM127131, U01 HG009088 to S.R.S.). 166 E.M.V.A further acknowledges support by the Phillip A. Sharp Innovation in Collaboration Award.

\section{Competing interests}

169 E.M.V. is a consultant for Tango Therapeutics, Genome Medical, Invitae, Foresite Capital, Dynamo, 170 and Illumina. E.M.V. received research support from Novartis and BMS, as well as travel support 171 from Roche and Genentech. E.M.V. is an equity holder of Syapse, Tango Therapeutics, Genome 172 Medical. 


\section{References}

176 1. Dees, N.D. et al. MuSiC: identifying mutational significance in cancer genomes. Genome Res 22, 1589-98 (2012).

Gonzalez-Perez, A. \& Lopez-Bigas, N. Functional impact bias reveals cancer drivers. Nucleic

2. Gonzalez-Perez, A. \& Lopez-B
Acids Res 40, e169 (2012).

3. Lawrence, M.S. et al. Mutational heterogeneity in cancer and the search for new cancerassociated genes. Nature 499, 214-218 (2013).

4. Martincorena, I. et al. Universal Patterns of Selection in Cancer and Somatic Tissues. Cell 171, 1029-1041 e21 (2017).

5. Weghorn, D. \& Sunyaev, S. Bayesian inference of negative and positive selection in human cancers. Nat Genet 49, 1785-1788 (2017).

6. Lawrence, M.S. et al. Discovery and saturation analysis of cancer genes across 21 tumour types. Nature 505, 495-501 (2014).

7. Hoadley, K.A. et al. Cell-of-Origin Patterns Dominate the Molecular Classification of 10,000 Tumors from 33 Types of Cancer. Cell 173, 291-304 e6 (2018).

8. Hofree, M. et al. Challenges in identifying cancer genes by analysis of exome sequencing data. Nat Commun 7, 12096 (2016).

9. Tokheim, C.J., Papadopoulos, N., Kinzler, K.W., Vogelstein, B. \& Karchin, R. Evaluating the evaluation of cancer driver genes. Proc Natl Acad Sci U S A 113, 14330-14335 (2016).

10. Guo, M.H. et al. Determinants of Power in Gene-Based Burden Testing for Monogenic Disorders. Am J Hum Genet 99, 527-539 (2016).

11. Lee, S., Abecasis, G.R., Boehnke, M. \& Lin, X. Rare-variant association analysis: study designs and statistical tests. Am J Hum Genet 95, 5-23 (2014).

12. Alexandrov, L.B. et al. Signatures of mutational processes in human cancer. Nature 500, 415-21 (2013).

13. Alexandrov, L.B. et al. Mutational signatures associated with tobacco smoking in human cancer. Science 354, 618-622 (2016).

14. Nik-Zainal, S. et al. Mutational processes molding the genomes of 21 breast cancers. Cell 149, 979-93 (2012).

15. Nik-Zainal, S. et al. Landscape of somatic mutations in 560 breast cancer whole-genome sequences. Nature 534, 47-54 (2016).

16. Ebrahimi, D., Alinejad-Rokny, H. \& Davenport, M.P. Insights into the motif preference of APOBEC3 enzymes. PLoS One 9, e87679 (2014).

17. Roberts, S.A. et al. Clustered mutations in yeast and in human cancers can arise from damaged long single-strand DNA regions. Mol Cell 46, 424-35 (2012).

18. Roberts, S.A. et al. An APOBEC cytidine deaminase mutagenesis pattern is widespread in human cancers. Nat Genet 45, 970-6 (2013).

19. Church, D.N. et al. DNA polymerase epsilon and delta exonuclease domain mutations in endometrial cancer. Hum Mol Genet 22, 2820-8 (2013).

20. Shinbrot, E. et al. Exonuclease mutations in DNA polymerase epsilon reveal replication strand specific mutation patterns and human origins of replication. Genome Res 24, 174050 (2014).

21. Goodman, M.F. \& Fygenson, K.D. DNA polymerase fidelity: from genetics toward a biochemical understanding. Genetics 148, 1475-82 (1998). 
219 22. Ganai, R.A. \& Johansson, E. DNA Replication-A Matter of Fidelity. Mol Cell 62, 745-55 (2016).

23. Chakravorty, D. et al. MYCbase: a database of functional sites and biochemical properties of Myc in both normal and cancer cells. BMC Bioinformatics 18, 224 (2017).

24. Izarzugaza, J.M., Redfern, O.C., Orengo, C.A. \& Valencia, A. Cancer-associated mutations are preferentially distributed in protein kinase functional sites. Proteins 77, 892-903 (2009).

25. Chang, M.T. et al. Identifying recurrent mutations in cancer reveals widespread lineage diversity and mutational specificity. Nat Biotechnol 34, 155-63 (2016).

26. Chang, M.T. et al. Accelerating Discovery of Functional Mutant Alleles in Cancer. Cancer Discov 8, 174-183 (2018).

27. Shiraishi, Y., Tremmel, G., Miyano, S. \& Stephens, M. A Simple Model-Based Approach to Inferring and Visualizing Cancer Mutation Signatures. PLoS Genet 11, e1005657 (2015).

28. Fredriksson, N.J. et al. Recurrent promoter mutations in melanoma are defined by an extended context-specific mutational signature. PLoS Genet 13, e1006773 (2017).

29. Varin, C., Reid, N. \& Firth, D. AN OVERVIEW OF COMPOSITE LIKELIHOOD METHODS. Statistica Sinica 21, 5-42 (2011).

30. North, B.V., Curtis, D. \& Sham, P.C. A note on the calculation of empirical P values from Monte Carlo procedures. Am J Hum Genet 71, 439-41 (2002).

31. Ewens, W.J. On estimating P values by the Monte Carlo method. Am J Hum Genet 72, 496-8 (2003).

32. Pearson, T.A. \& Manolio, T.A. How to interpret a genome-wide association study. JAMA 299, 1335-44 (2008).

33. Ainscough, B.J. et al. DoCM: a database of curated mutations in cancer. Nat Methods 13, 806-7 (2016).

34. Forbes, S.A. et al. COSMIC: exploring the world's knowledge of somatic mutations in human cancer. Nucleic Acids Res 43, D805-11 (2015).

35. Futreal, P.A. et al. A census of human cancer genes. Nat Rev Cancer 4, 177-83 (2004).

36. Glozak, M.A. \& Seto, E. Histone deacetylases and cancer. Oncogene 26, 5420-32 (2007).

37. Li, Y. \& Seto, E. HDACs and HDAC Inhibitors in Cancer Development and Therapy. Cold Spring Harb Perspect Med 6(2016).

38. Ropero, S. \& Esteller, M. The role of histone deacetylases (HDACs) in human cancer. Mol Oncol 1, 19-25 (2007).

39. Clark, V.E. et al. Recurrent somatic mutations in POLR2A define a distinct subset of meningiomas. Nat Genet 48, 1253-9 (2016).

40. Liu, Y. et al. TP53 loss creates therapeutic vulnerability in colorectal cancer. Nature 520, 697-701 (2015).

41. Reich, M. et al. GenePattern 2.0. Nat Genet 38, 500-1 (2006).

42. Burli, R.W. et al. Design, synthesis, and biological evaluation of potent and selective class IIa histone deacetylase (HDAC) inhibitors as a potential therapy for Huntington's disease. $J$ Med Chem 56, 9934-54 (2013).

43. Maolanon, A.R., Madsen, A.S. \& Olsen, C.A. Innovative Strategies for Selective Inhibition of Histone Deacetylases. Cell Chem Biol 23, 759-768 (2016). 
a passenger

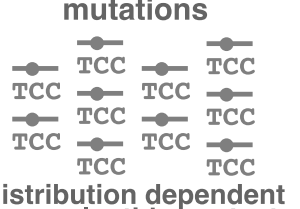
distribution dependent
on nucleotide context

$$
\begin{gathered}
\text { driver } \\
\text { mutations } \\
\text { accumulation in } \\
\text { functional positions }
\end{gathered}
$$

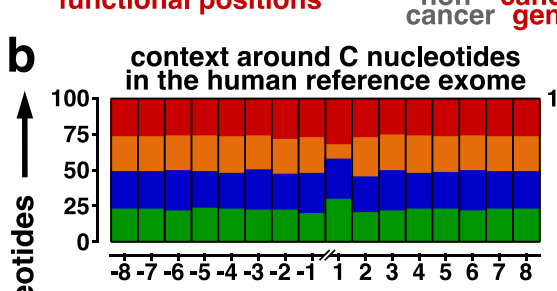

context around $\mathrm{C}>\mathrm{T}$

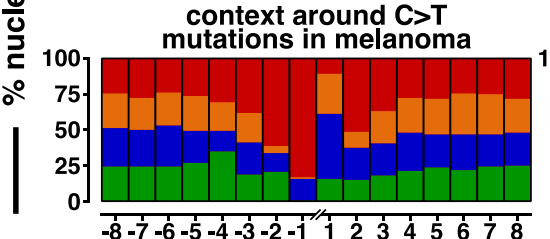

$-8-7-6-5-4-3-2-1 \quad 125345678$ detection of cancer genes based on mutation counts and nucleotide context
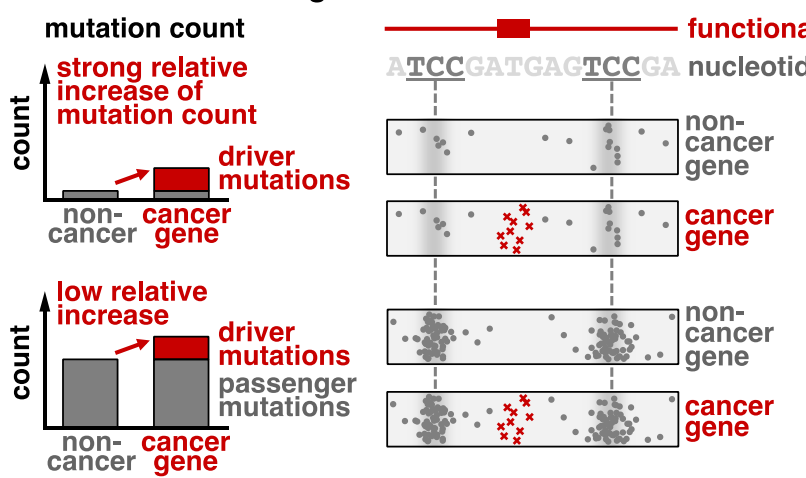

context around $\mathrm{C}>\mathrm{G}$

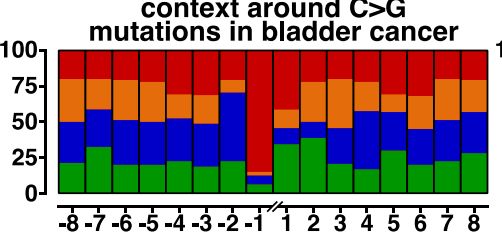

context around $\mathrm{C}>\mathrm{T}$

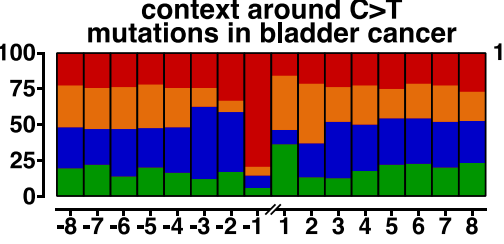

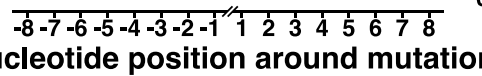

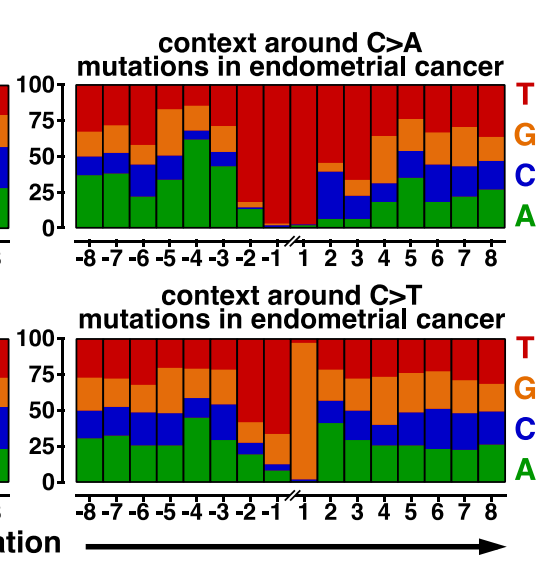

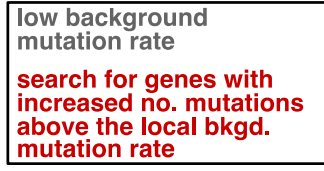

mutation rate

high background mutation rate

search for genes with increased no mutations in an unusual context
C trinucl. $\boldsymbol{A}_{\text {context }} \rightarrow \begin{gathered}\text { original } \\ \text { context }\end{gathered}$
$\mathbf{e} \begin{aligned} & \text { broader } \\ & \text { nucl. context }\end{aligned} \boldsymbol{\Lambda}_{\text {context }}$
$\rightarrow$ original likelihood
nuci $\downarrow \downarrow \downarrow$ composite context $\boldsymbol{\Lambda}_{-1} \times \boldsymbol{\lambda}_{0} \times \lambda_{1} \rightarrow$ likelihood

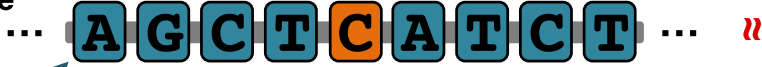

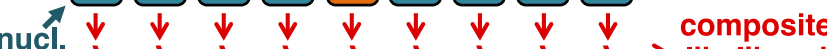 context $\boldsymbol{\lambda}_{-4} \times \boldsymbol{\lambda}_{-3} \times \boldsymbol{\lambda}_{-2} \times \boldsymbol{\lambda}_{-1} \times \boldsymbol{\lambda}_{0} \times \boldsymbol{\lambda}_{1} \times \boldsymbol{\lambda}_{2} \times \boldsymbol{\lambda}_{3} \times \boldsymbol{\lambda}_{4} \rightarrow$ likelihood

\section{T
G
C
A
T
C
A}

d correlation across 96 trinucl. contexts $O C>A O C>G \quad C>T$ OT $>$ A OT $>$ C OT $>$ G

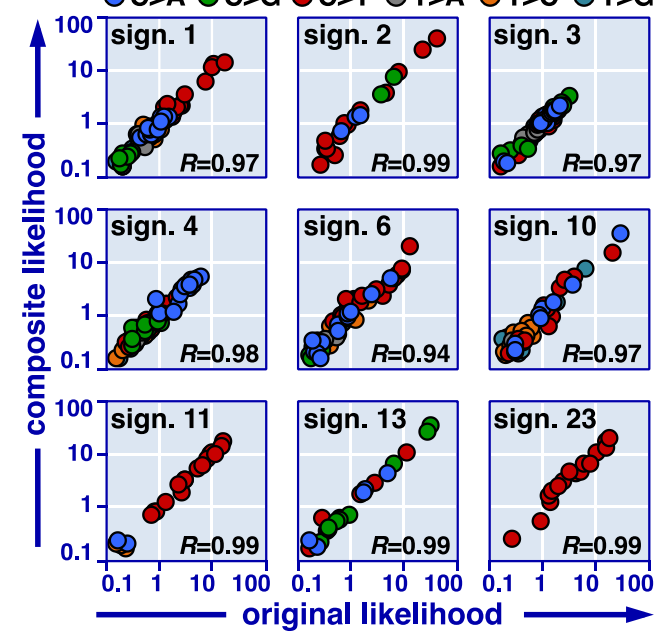

f correlation across $2.2 \times 10^{5}$ nucleotide contexts density of data points: high $\square \square \square \square \square$ low

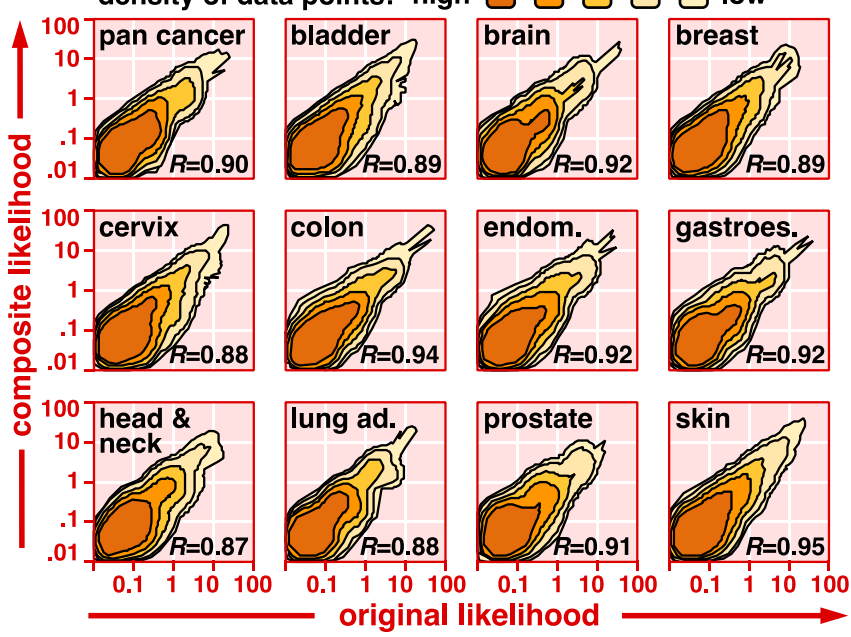


Fig. 1 | A biologically informed statistical framework to discover candidate cancer genes. a,

265 Schematic of our statistical framework to discover candidate cancer genes based on nucleotide

266 context. Passenger mutations accumulate in characteristic nucleotide contexts (gray, left), 267 whereas driver mutations typically accumulate in functionally relevant positions (red, left). We searched for genes harboring an increased number of nonsynonymous mutations above the local background mutation rate (mutational recurrence, middle). Further, we searched for genes with an excess of mutations in nucleotide contexts that deviate from the characteristic nucleotide context around passenger mutations (mutations in unusual contexts, right). In tumors with high background mutation rates, the second criterion allowed us to actively suppress mutations in the test statistics that were likely to be passenger mutations based on their surrounding nucleotide context (gray). b, The nucleotide context around passenger mutations is visualized for three cancer types with high average background mutation rates. In brief, we counted how often we observed which nucleotide in the context around recurrent passenger mutations ( \pm 8 nucleotides). These plots show that the flanking 5' and 3' nucleotides have the strongest impact on the local mutation probability ( \pm 1 , trinucleotide context). However, also flanking nucleotides outside of the trinucleotide context have a substantial impact on the local mutation probability, suggesting that the broad nucleotide context around passenger mutations contains a relevant biological signal that we needed to consider in our approach. c-f, To integrate this signal into our statistical framework, we developed a composite likelihood model that characterizes the broad context around passenger mutations. c, Mutation probabilities of trinucleotide contexts are commonly modeled by determining the mutation probability of each possible trinucleotide context independently11-14 (original likelihood, top). Instead, we integrated the effect of the flanking $5^{\prime}$ and 3 ' nucleotides, as well as the base substitution type as independent factors into a composite likelihood model (bottom). d, For each classical trinucleotide mutation signature ${ }^{11-14}$, we plotted the original mutational likelihood (x-axis) against the composite likelihood (y-axis). Dot colors reflect the six different base substitution types, and Pearson correlations are annotated on the bottom right. These analyses revealed that mutation probabilities of trinucleotide contexts could be decomposed into the effects of their central and flanking $5^{\prime}$ and $3^{\prime}$ nucleotides, thus corroborating the validity of our composite likelihood approach for trinucleotide contexts. e, We next generalized the composite likelihood model to broader nucleotide contexts. In parallel to our approach for trinucleotide contexts, we integrated the effect of each flanking nucleotide in the 
295 broad context as an independent and multiplicative factor into the composite likelihood. f, We 296 then counted the number of mutations in each possible 7-nucleotide context (x-axis, original 297 likelihood) and compared them with the composite likelihood (y-axis). Since the number of 298 possible nucleotide contexts was too large to be visualized directly, we plotted the data point 299 density. Similar plots for the remaining trinucleotide signatures and cancer types are shown in 300 Figures S7a-c. An analysis of the contribution of flanking nucleotides outside of the trinucleotide 301 context to the local mutation probability in the composite likelihood model is shown in Figures 302 S7d and S8. 


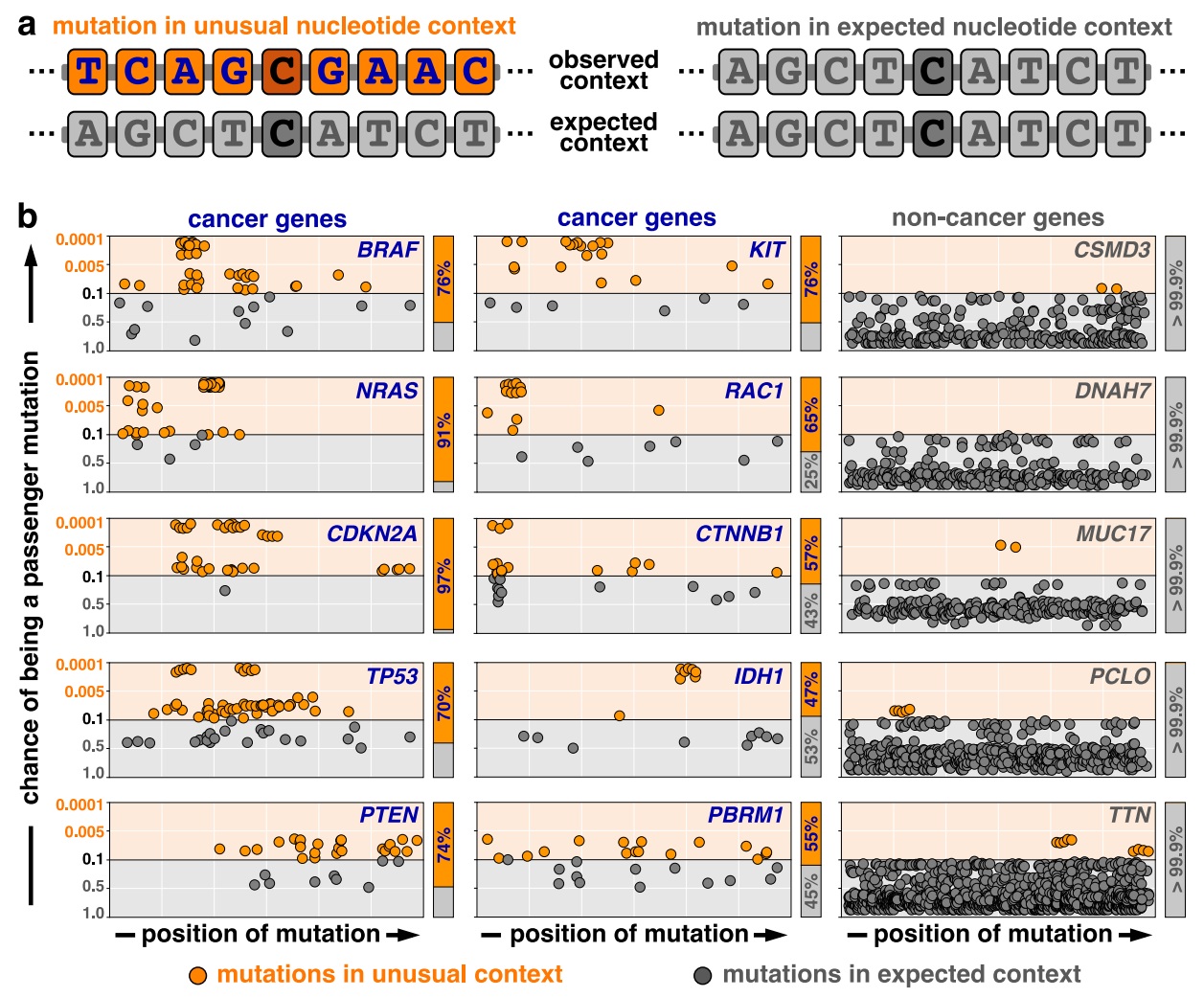

Fig. 2 | Cancer driver genes harbor an excess of mutations in unusual nucleotide contexts. a,

307 For each mutation, we compared its nucleotide context (observed context, top) with the characteristic context around passenger mutations (expected context, bottom). We derived a probability score that indicated whether the mutation occurred in an unusual (left, orange) or expected (right, gray) nucleotide context (Methods). b, We corrected these probabilities for multiple hypothesis testing (false-discovery rates, y-axis) and plotted them against their genomic position ( $\mathrm{x}$-axis). In cancer genes a substantial number of mutations occurred in unusual sequence contexts (left, middle). In non-cancer genes mutations in unusual sequence contexts were extremely rare (right). This suggested that cancer driver genes harbor an increased number of mutations in unusual nucleotide contexts that deviate from the characteristic nucleotide context around passenger mutations. This observation provides a novel biological criterion to discriminate between driver and passenger mutations. 

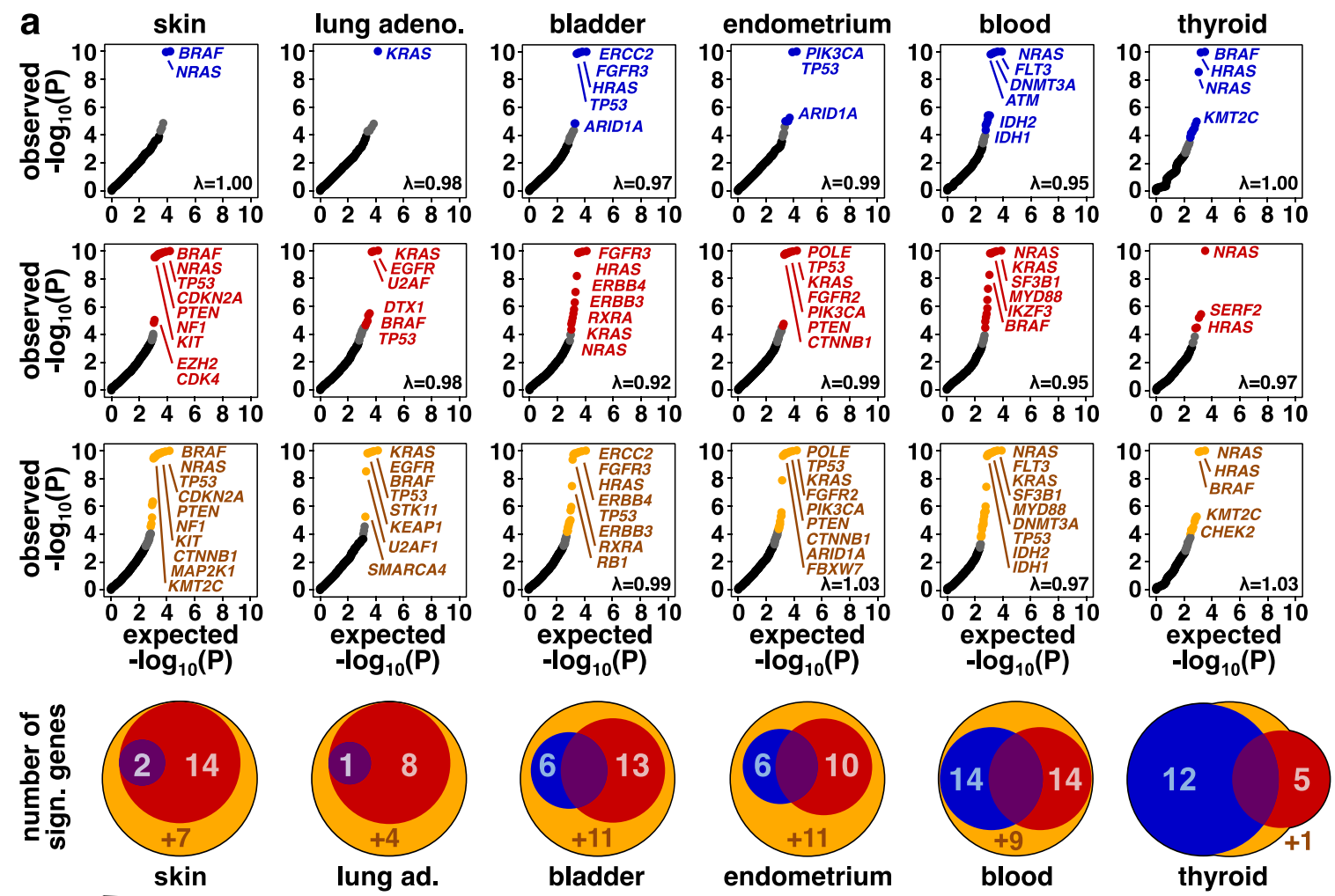

\section{high \\ significance based on
mutational recurrence}

background mutation rate

low

significance based on

nucleotide context

significance based on
combining both criteria
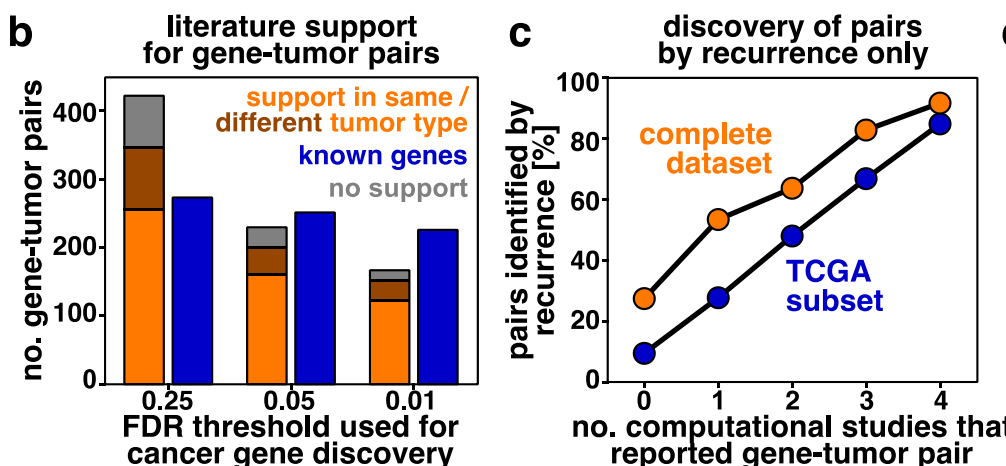

no. computational studies that reported gene-tumor pair
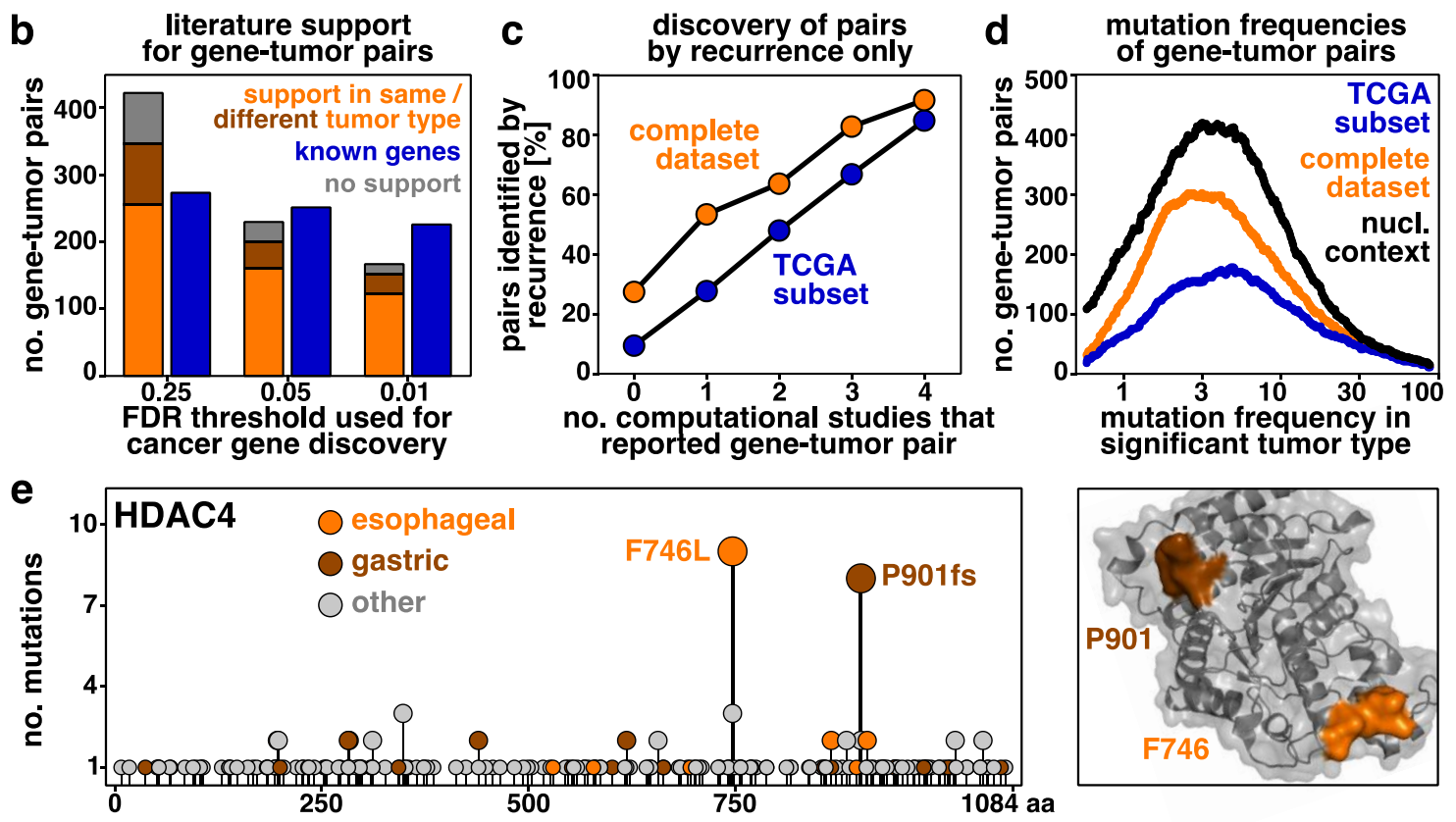
Fig. 3 | Discovery and characterization of candidate cancer genes identified based on

324 nucleotide context. a, We determined which genes emerged as significantly mutated (false325 discovery rate, FDR $<0.25$ ) based on their mutational recurrence (blue) and based on their excess 326 of mutations in "unusual" nucleotide contexts (red). Further, we identified candidate cancer genes

327 based on a statistical model that combined mutational recurrence and nucleotide context 328 (orange). We compared the expected (x-axis) and observed (y-axis) $p$-values derived from these 329 three statistical models using Q-Q-plots. Venn diagrams visualize the overlap in significant genes 330 detected with these three models (bottom). These analyses revealed that increased mutation 331 counts and unusual nucleotide contexts provide two complementary criteria for the discovery of 332 cancer genes. Integrating both aspects into a combined significance model enabled discovery of 333 candidate cancer genes across tumor types with high and low background mutation rates (left to 334 right). b, We stratified our findings based on their support in the literature. Known gene-tumor pairs, which had been reported as significantly mutated previously, are colored in blue. Novel gene-tumor pairs, which had not been reported as significantly mutated previously, are colored in orange (experimental support in the same tumor type), brown (literature support in a different tumor type), or gray (no support). For rigorous FDR thresholds (FDR<0.01), a majority of the significant gene-tumor pairs (82\%, 323/395) involved canonical cancer genes in the Cancer Gene Census 34,35 . Further, most gene-tumor pairs had been known previously or had experimental literature support in the same tumor type (89\%, 351/395 for FDR<0.01). For less stringent FDR thresholds, the absolute number of novel findings with experimental literature support increased, and the number of findings without literature support $(11 \%, 75 / 697)$ did not exceed the expected false-discovery rate $(\mathrm{FDR}<0.25)$. $\mathbf{c}$, We counted for each gene-tumor pair $(\mathrm{FDR}<0.25)$ how many previous studies reported the gene-tumor pair as significantly mutated $4,6,7,33$ ( $\mathrm{x}$-axis). Further, we examined whether the gene-tumor pair was also identified using an established recurrence-based approach $^{3}$ (y-axis). The concordance between these two measures potentially reflects the fact that most previous pan-cancer gene discovery studies used recurrence-based approaches to identify cancer genes ${ }^{4,6,7,33}$. d, We explored the mutation frequencies of the gene-tumor pairs that emerged as significantly mutated based on their recurrence in the TCGA subset (blue), in the complete dataset (orange), or when additionally considering the nucleotide context around mutations (black). This density plot revealed that both the addition of 4,913 samples from TCGAindependent studies and considering the nucleotide context around mutations independently 
354 contributed to the discovery of rare candidate cancer genes. e, Exemplary evidence for the 355 candidate cancer gene HDAC4. Left: The distribution of HDAC4 mutations is visualized as a needle 356 plot. For each amino acid substitution the number of samples (y-axis) is plotted against its 357 position in the peptide sequence (x-axis). Dot colors reflect the tumor types, in which the amino 358 acid substitution was detected. Right: The position of the two mutational hotspots is visualized 359 using a crystal structure 42 (PDB: 4CBY). A previous study reported a hydrogen bond and salt 360 bridge network between W762, E764, and R730, which along with F746 form a closed 361 hydrophobic patch peripheral to the catalytic center of HDAC443 (orange). Evidence for other 362 candidate cancer genes can be found in Figures S11-S13. 

under aCC-BY-NC-ND 4.0 International license.

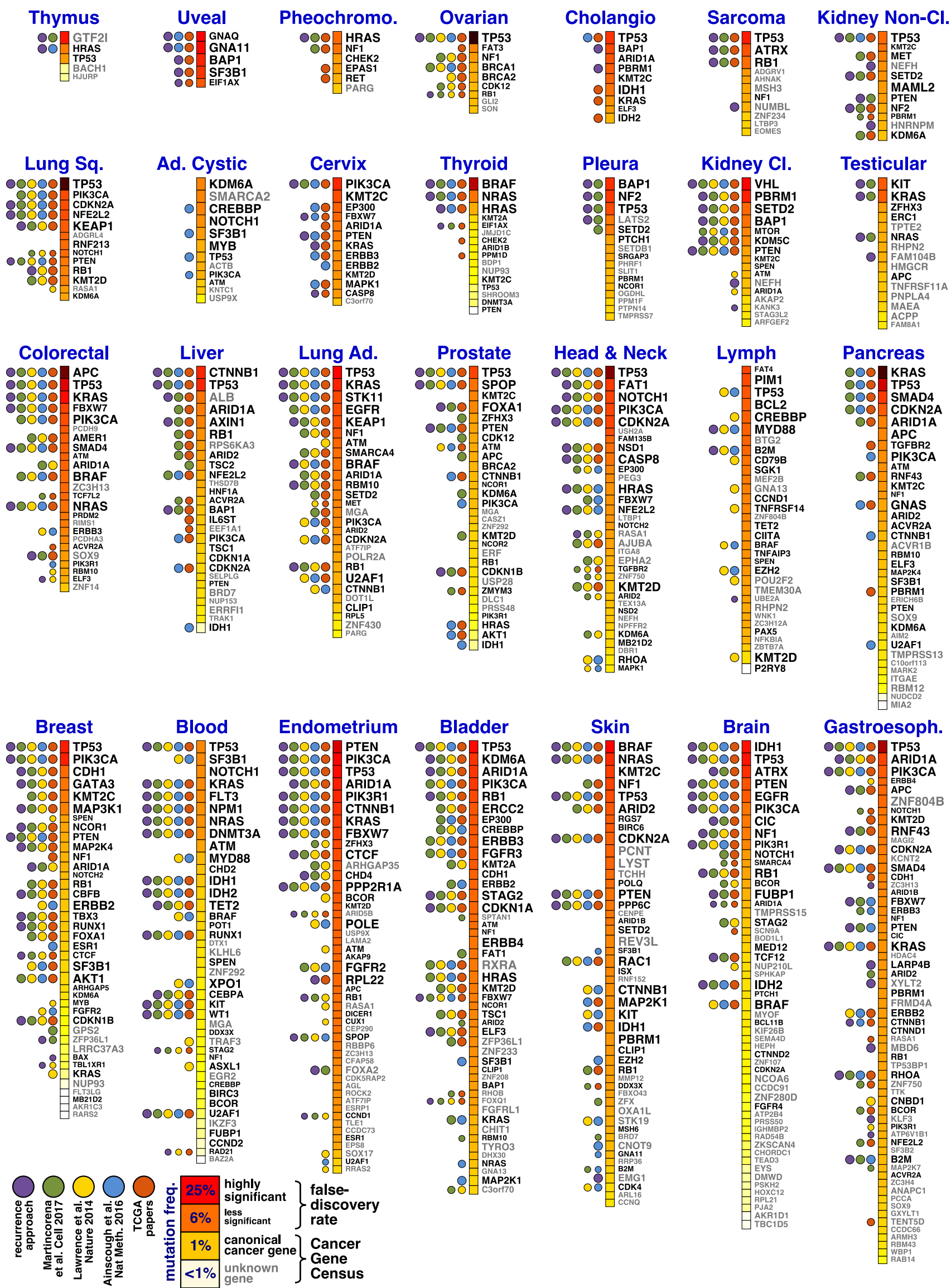


365 Fig. 4 | A refined catalog of driver genes involved in human cancer. We applied our statistical 366 framework to whole-exome sequencing data from 12,004 tumors. Significant gene-tumor pairs 367 (FDR $<0.25)$ are listed in decreasing order according to their mutation frequency, which is 368 reflected by the color of the square next to the gene name (dark red to white). The font size of the 369 gene name reflects its significance (false-discovery rate), and the font color (black vs. white) 370 indicates whether the gene is a canonical cancer gene in the Cancer Gene Census ${ }^{34,35}$. To determine 371 which gene-tumor pairs had been known previously, we benchmarked our results against all 372 TCGA marker papers ${ }^{7}$ (orange), a meta-analysis ${ }^{33}$ of 876 publications (blue), the tumorportal 373 database $^{6}$ (yellow), and a pan-cancer study, which adopted the $\mathrm{dN} / \mathrm{dS}$ ratio for cancer gene 374 discovery $^{4}$ (green). We further ran an established recurrence-based approach ${ }^{3}$ on our dataset 375 (purple) to determine which gene-tumor pairs were identified based on recurrence alone. A more 376 detailed overview of the driver mutation landscape of individual tumor types is provided in 377 Figures S18-S45. An interactive visualization of these results can be found online (www.cancer378 genes.org). 


\begin{tabular}{|c|c|c|c|c|c|c|c|c|}
\hline & \multicolumn{2}{|c|}{ FDR < 25\% } & \multicolumn{2}{|c|}{ FDR $<10 \%$} & \multicolumn{2}{|c|}{ FDR $<5 \%$} & \multicolumn{2}{|c|}{ FDR < 1\% } \\
\hline & Genes & G-T Pairs & Genes & G-T Pairs & Genes & G-T Pairs & Genes & G-T Pairs \\
\hline Total & 379 & 697 & 298 & 562 & 252 & 484 & 201 & 395 \\
\hline Known & 127 & 274 & 123 & 262 & 121 & 253 & 114 & 227 \\
\hline Novel & 252 & 423 & 175 & 300 & 131 & 231 & 87 & 168 \\
\hline $\begin{array}{l}\text { support in } \\
\text { same type }\end{array}$ & 110 & 257 & 85 & 198 & 70 & 161 & 50 & 124 \\
\hline $\begin{array}{l}\text { support in } \\
\text { different typ }\end{array}$ & pe 69 & 91 & 44 & 55 & 31 & 40 & 21 & 28 \\
\hline - no support & 73 & 75 & 46 & 47 & 30 & 30 & 16 & 16 \\
\hline
\end{tabular}

381 Table 1 | Stratification of candidate cancer genes by literature support. To examine the 382 biological relevance of our findings, we stratified them based on their literature support. Genes 383 and gene-tumor (G-T) pairs that had been reported as significantly mutated in at least two 384 previous computational studies were classified as known (blue, 2nd row). Novel genes and gene385 tumor pairs, which had not been reported as significantly mutated previously (red, 3rd row), were 386 further stratified depending on whether there was literature support (experimental or clinical) for 387 the same tumor type in which we discovered them as significantly mutated (orange, 4th row), 388 supporting literature for a different tumor type (brown, 5th row), or no supporting data (gray, 6th 389 row). Depending on their literature support level, 94\% (known, 257/274), 72\% (same tumor type, 390 186/257), 23\% (different tumor type, 21/91), and 3\% (no support, 2/75) of the gene-tumor pairs 391 (FDR<0.25) involved canonical cancer genes present in the Cancer Gene Census ${ }^{34,35}$, compared 392 with a rate of $3.8 \%$ for random gene-tumor pairs. Thus, literature support levels provide a 393 measure to prioritize our findings based on their external validity. 